\title{
DISTINGUISHING CAP AND CORE CONTRIBUTIONS TO THE PHOTOCONDUCTIVE TERAHERTZ RESPONSE OF SINGLE GaAs BASED CORE-SHELL-CAP NANOWIRE DETECTORS
}

\author{
K. Peng a, b , P. Parkinson ' ${ }^{\text {, }}$ L. Fu ${ }^{\text {a }}$, Q. Gao ${ }^{\text {a }}$, J. Boland ${ }^{\text {b }, ~ Y .-N . ~ G u o ~ a ~, ~ N . ~ J i a n ~}{ }^{\text {a }}$, H.H. Tan ${ }^{\text {a }}$, \\ M.B. Johnston ${ }^{\mathrm{b}}$, and C. Jagadish ${ }^{\mathrm{a}}$ \\ ${ }^{a}$ Department of Electronic Materials Engineering, Research School of Physics and Engineering, The Australian National \\ University, Canberra, ACT 2601, Australia \\ ${ }^{\mathrm{b}}$ Department of Physics, University of Oxford, Clarendon Laboratory, Parks Road, Oxford OX1 3PU, United Kingdom \\ ' School of Physics and Astronomy and the Photon Science Institute, University of Manchester, \\ Manchester M13 9PL, United Kingdom \\ Email: kun.peng@physics.ox.ac.uk
}

Received 1 February 2018; accepted 22 March 2018

\begin{abstract}
GaAs nanowires are promising candidates for advanced optoelectronic devices, despite their high surface recombination velocity and large surface-area-to-volume ratio, which renders them problematic for applications that require efficient charge collection and long charge-carrier lifetimes. Overcoating a bare GaAs nanowire core with an optimized larger-bandgap AlGaAs shell, followed by a capping layer of GaAs to prevent oxidation, has proven an effective way to passivate the nanowire surface and thereby improve electrical properties for enhanced device performance. However, it is difficult to quantify and distinguish the contributions between the nanowire core and cap layer when measuring the optoelectronic properties of a nanowire device. Here, we investigated the photoconductive terahertz $(\mathrm{THz})$ response characteristics of single GaAs/AlGaAs/GaAs core-shell-cap nanowire detectors designed for THz time-domain spectroscopy. We present a detailed study of the contributions of the GaAs cap layer and GaAs core on the ultrafast optoelectronic performance of the detector. We show that both the GaAs cap and core contribute to the photoconductive signal in proportion to their relative volume in the nanowire. By increasing the cap volume ratio to above $90 \%$ of the total GaAs volume, a quasi-direct-sampling type photoconductive nanowire detector can be achieved that is highly desirable for low-noise and fast data acquisition detection.
\end{abstract}

Keywords: GaAs, nanowire devices, ultrafast spectroscopy

PACS: 81.05.Ea, 81.07.Gf, 85.60.Bt, 07.57.Kp, 78.47.jh

\section{Introduction}

Spectroscopy and imaging with pulsed terahertz $(\mathrm{THz})$ radiation have been demonstrated as powerful tools for materials characterization and identification [1-3], non-destructive inspection [4], safety scans for security [5] and medical examination [6, 7]. THz photoconductive antennas (based on Auston switches [8]) have been developed over the last 30 years and have now established themselves as reliable and effective detection elements used in many commercial pulsed $\mathrm{THz}$ systems, including $\mathrm{THz}$ time-domain spectroscopy (THzTDS) systems [9]. Optimized photoconductive materials are essential for developing photoconductive antennas with high sensitivity and broadband performance. Thus materials for photoconductive antennas have been thoroughly studied 
over the years, from the first demonstration of Arion irradiated crystalline $\mathrm{Si}$ epitaxially grown on sapphire [8, 10] to semi-insulating GaAs [11], low temperature-grown (In)GaAs [12, 13] and ionimplanted InP [14]. However, the main challenge of synthesizing and selecting photoconductive materials still persists. For good device performance, these materials require specific optoelectronic properties: a very low carrier lifetime (to lower the noise and produce a direct or integrating detector sampling mode); reasonable carrier mobility (to ensure a high or measurable signal); appropriate bandgap (for device to be operational); and high material dark resistivity (to minimize the noise current).

It is well known that the carrier lifetimes in core-only GaAs nanowires are extremely short [15], of the order of picoseconds, owing to their high surface recombination velocity [16, 17]. These short lifetimes lead to large surface scattering and poor charge collection, resulting in poor performance for optoelectronic applications. Some studies have shown that the minority carrier lifetime in GaAs based nanowires can be tuned over the range of around 1 to 1600 ps through careful control of the crystal quality [15, 18, 19], doping [20-22] and surface passivation via AlGaAs shell growth [18]. These advances have allowed the design and growth of GaAs based nanowires with tailored properties for optimal photoconductive $\mathrm{THz}$ detection. In 2014, we successfully demonstrated the single GaAs/ AlGaAs/GaAs core-shell-cap nanowire photoconductive detectors [23, 24], incorporated into a standard THz-TDS system as a microscopic sensor. The single nanowire detectors showed a good signal-to-noise ratio when compared with traditional detectors including ion-implanted photoconductive antennas and electro-optic crystals. Due to the nanoscale size and fabrication on an insulating substrate, the nanowire detectors exhibited a largely reduced dark current and low-noise nature. This relaxed the requirement on the carrier lifetime of its photoconductive material from the previously required picosecond to nanosecond (for integrating the sampling mode), widely extending the availability of nanowire material systems for $\mathrm{THz}$ photoconductive detection.

We also noted an additional signal at the earliest time (a very fast decay at the first 25 ps af- ter photoexcitation) when measuring photoconductivity lifetimes for these GaAs/AlGaAs/GaAs core-shell-cap nanowires [24, 25], which cannot be observed for bare GaAs nanowires [16] or GaAs/AlGaAs core-shell nanowires [25]. This result suggests that the GaAs cap has a significant influence on the photoconductivity properties of the nanowires, rather than solely preventing the AlGaAs shell from oxidation [23]. After a further study [25], we confirmed that the early rapid decay signal is related to the fast electron trapping at the GaAs cap surface for the carriers photogenerated in the GaAs cap. Furthermore, we found that the GaAs/AlGaAs/GaAs core-shellcap nanowires exhibit higher electron mobilities than the core-shell nanowires because the GaAs cap layer also plays a role in spatially separating the carriers in the core from the nanowire surface leading to improved mobility (in the same way as the AlGaAs shell does). Therefore, it is necessary to isolate the cap and core contributions in the GaAs/AlGaAs/GaAs core-shell-cap nanowire photoconductive detector for a complete understanding of how it works. In this work, we designed the GaAs cap and the GaAs core to be comparable in their volume but with a huge difference in photoconductivity lifetimes. The photoconductivity lifetime determines the device sampling mode [26] and hence the analytic approach required in data analysis. Given the considerable difference in the photoconductivity lifetime of the GaAs cap and the GaAs core, two sampling modes occurred in the single GaAs/AlGaAs/GaAs core-shell-cap nanowire detector simultaneously, which are distinguishable via analysis of its $\mathrm{THz}$ response characteristics. This work provides a useful guide for the future design of ultrafast optoelectronic devices with GaAs based core-shellcap nanowires.

\section{Experiment}

The GaAs/AlGaAs/GaAs core-shell-cap nanowires were grown on semi-insulating GaAs (111) B substrates by the metalorganic chemical vapour deposition (MOCVD) technique via the vapourliquid-solid (VLS) mechanism [23]. The growth was realized in a temperature-controlled reactor at a pressure of $100 \mathrm{mbar}$ and a total gas flow rate of $15 \mathrm{l} / \mathrm{min}$. Trimethylgallium (TMG), 
trimethylaluminium (TMAl) and arsine $\left(\mathrm{AsH}_{3}\right)$ were used as the $\mathrm{Ga}, \mathrm{Al}$ and As source materials, respectively. Au nanoparticles of $150 \mathrm{~nm}$ in diameter were used as catalysts and dispersed onto the GaAs substrates prior to growth. The substrates with $\mathrm{Au}$ nanoparticles were annealed at $600^{\circ} \mathrm{C}$ for $10 \mathrm{~min}$ under $\mathrm{AsH}_{3}$ ambient to desorb surface contaminants prior to nanowire growth. After cooling to the desired temperature, TMG flow was switched on to initiate nanowire growth. The GaAs core was grown by a two-temperature procedure [19], which has significant advantages in minimizing undesirable tapering and eliminating twin defects to provide a high-quality uniformdiameter GaAs core, resulting in enhanced carrier mobility and lifetime of the nanowire. The growth was initiated at $450^{\circ} \mathrm{C}$ with a $1 \mathrm{~min}$ "nucleation" step followed by a prolonged $(120 \mathrm{~min})$ growth at $375^{\circ} \mathrm{C}$. Source flows of TMG and $\mathrm{AsH}_{3}$ were $1.2 \times 10^{-5}$ and $5.4 \times 10^{-4} \mathrm{~mol} / \mathrm{min}$, respectively. After the GaAs core growth, TMG was switched off and the growth temperature was ramped to $750^{\circ} \mathrm{C}$ in $\mathrm{AsH}_{3}$ ambience. An AlGaAs shell was then grown for $3 \mathrm{~min} 45 \mathrm{~s}$ at this temperature according to the optimized procedure previously developed [23]. Finally, the nanowires were terminated by GaAs cap layer growth using a fast one-temperature growth technique [19] for $1 \mathrm{~min}$ at the same temperature, to produce a thin defective cap layer with a very short charge-carrier lifetime in it and a high carrier recombination velocity at the cap surface. The morphologies of the GaAs/AlGaAs/ GaAs nanowires were characterized using scanning electron microscopy (SEM), and their coreshell-cap structure was studied by transmission electron microscopy (TEM). Figure 1(a) shows that the GaAs/AlGaAs/GaAs nanowires have a core-shell-cap structure with a 150 -nm diameter GaAs core, an AlGaAs shell of $\sim 28 \mathrm{~nm}$ and a thin GaAs cap layer of $\sim 35 \mathrm{~nm}$, with an average length of around $13 \mu \mathrm{m}$.

A time-domain optical-pump $\mathrm{THz}$-probe (OPTP) spectroscopy system [16, 20, 22] was used to measure the photoconductivity lifetime of an ensemble of GaAs/AlGaAs/GaAs nanowires. This photoconductivity lifetime determines the detector operation type (direct or integrating sampling) [26] and the signal processing technique required to recover the $\mathrm{THz}$ electric field from the current measured in the photoconductive detector. More details of this measurement can be found in Refs. [27, 28]. Ultrafast photoexcitation at $800 \mathrm{~nm}(1.55 \mathrm{eV})$ was employed to selectively excite only the GaAs in the nanowires. It was shown that the carrier decay was not single-exponential for the $\mathrm{GaAs} / \mathrm{AlGaAs} / \mathrm{GaAs}$ nanowires, including an initial rapid decay $\left(\tau_{\mathrm{f}} \sim\right.$ a few ps) due to fast electron trapping at the un-passivated GaAs cap layer surface, and then slower decay $\left(\tau_{\mathrm{s}}=4600 \mathrm{ps}\right)$ due to recombination in the GaAs core [25]. It is worth noting that the fast decay is only present in the GaAs nanowire sample with the GaAs cap [17,
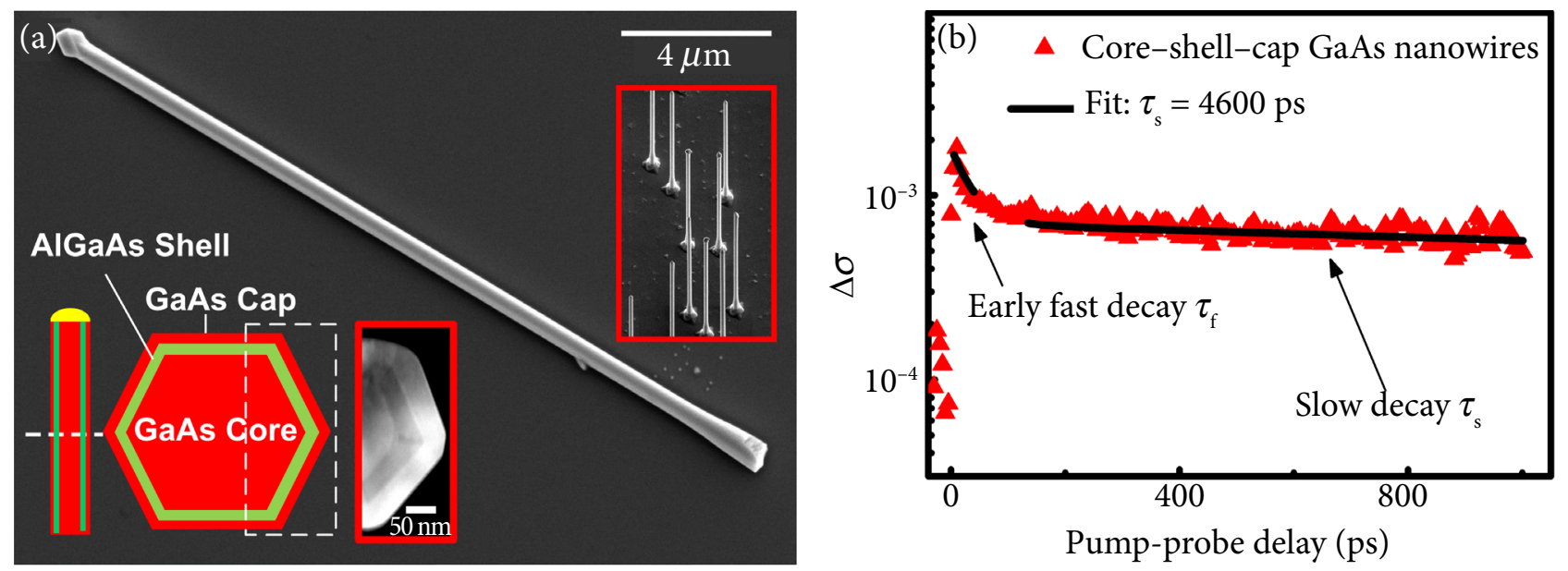

Fig. 1. (a) SEM images of the single GaAs/AlGaAs/GaAs core-shell-cap nanowire used in this study (inset images also include a SEM image of nanowires grown on wafer, a schematic cross-section, a cross-sectional TEM image of the nanowire). (b) OPTP photoconductivity decay of the ensemble of core-shell-cap GaAs nanowires in (a) (solid black lines are two-exponential fit to the data. The $\tau_{\mathrm{s}}$ shows the dominant carrier lifetime for the sample). 
25] which is a clear indication that the origin of the fast decay is the cap.

To fabricate the single nanowire detectors, the as-grown nanowire substrate was cleaved into small pieces and placed in an isopropyl alcohol solution for a 30-second ultra-sonication to transfer the nanowires into the solution. The solution was dropped onto $z$-cut quartz substrates (which are $\mathrm{THz}$ transparent) and allowed to dry in air at room-temperature. The substrates were spin-coated with photoresist and patterned using conventional ultraviolet (UV) photolithography. An oxygen plasma descum process was used for further removal of the photoresist residue on the nanowire, followed by $4 \% \mathrm{HCl}$ chemical etching to remove the native oxide layer from the nanowire surface (the GaAs cap surface). The detector structures were achieved by using electron beam evaporation to deposit $\mathrm{Ti} / \mathrm{Au}(10 / 300 \mathrm{~nm})$ on the electrode patterns. The geometry for bowtie single GaAs/AlGaAs/GaAs nanowire $\mathrm{THz}$ detectors used in this study is shown in Fig. 2(a). (a)

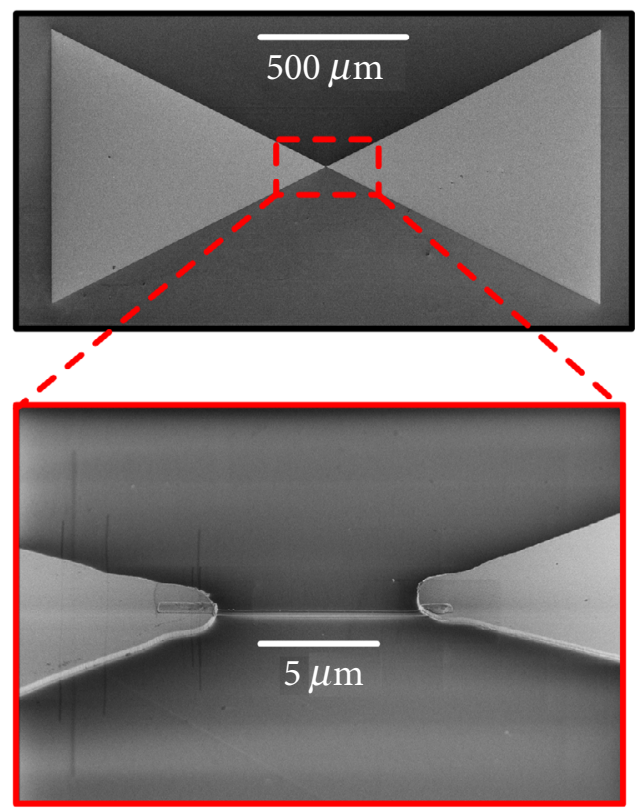

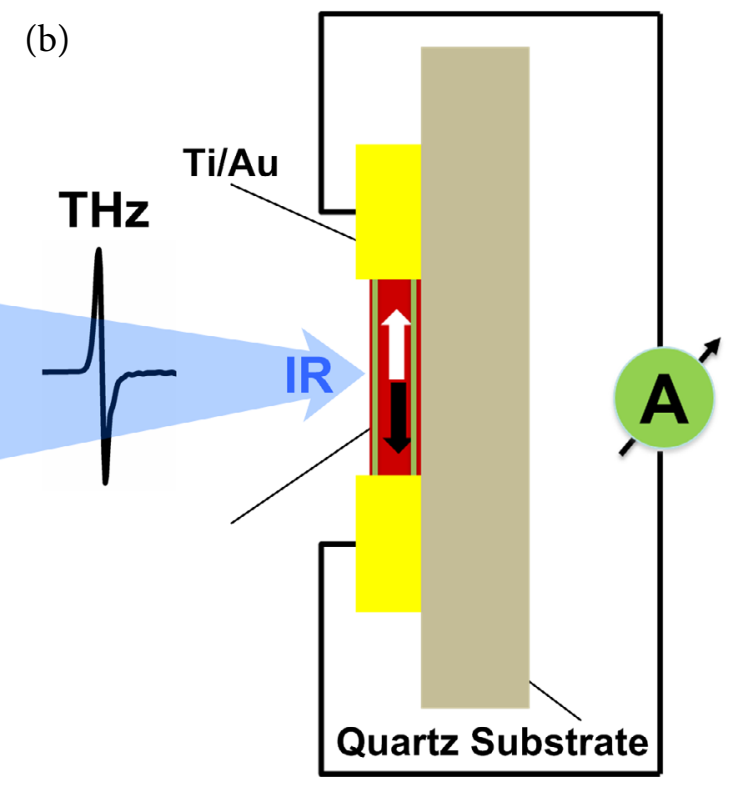

(c)

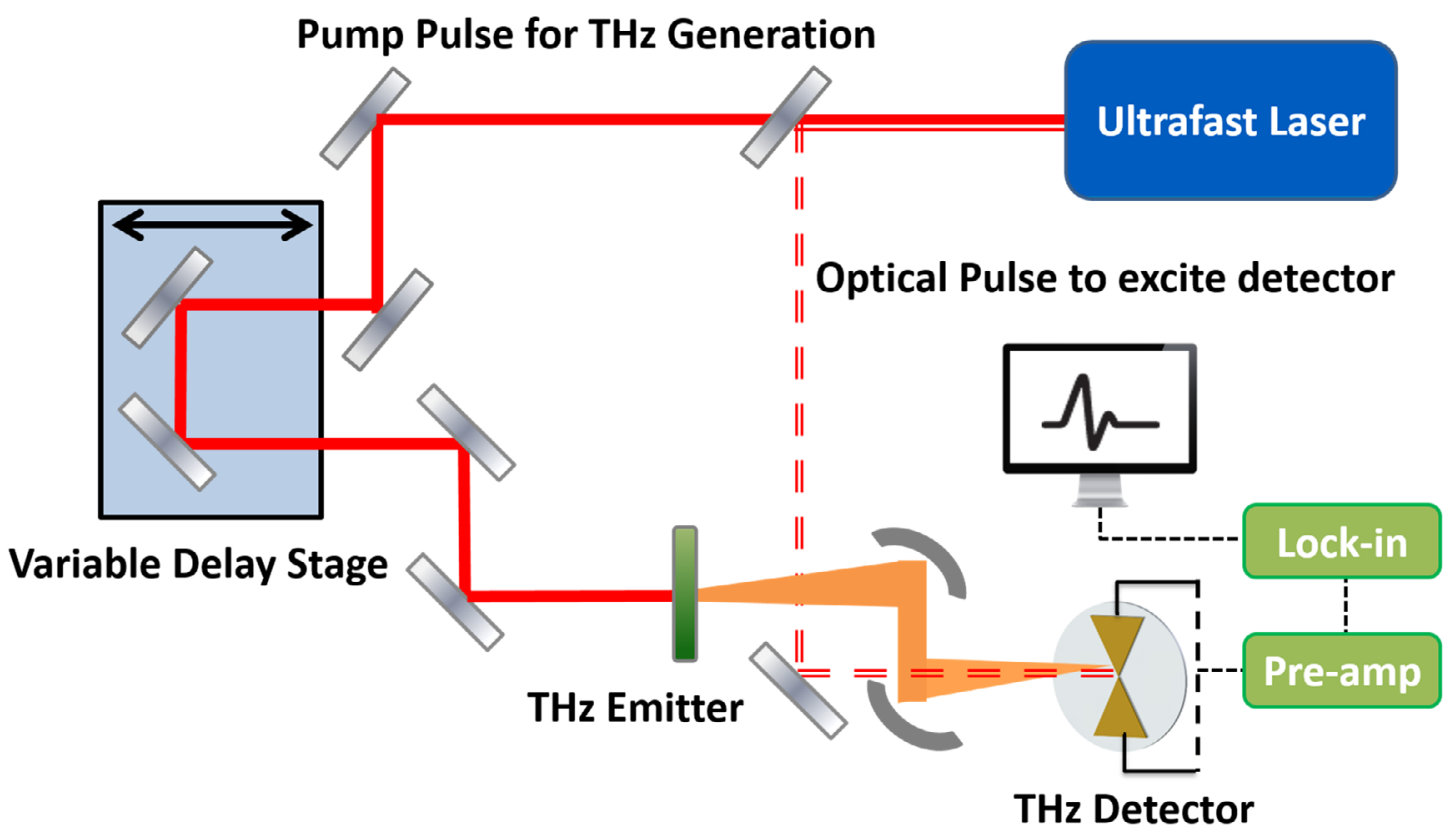

Fig. 2. (a) SEM images of the contacted GaAs/AlGaAs/GaAs core-shell-cap nanowire under low (upper) and high (lower) magnifications. (b) A schematic diagram of a photoconductive single nanowire detector in operation. (c) A schematic diagram of the THz-TDS system used for this work. 
Detailed dimensions of the bow-tie electrode geometry can be found in Ref. [29]. The electrode gap was designed to be $5 \mu \mathrm{m}$ on the photomask but was measured as $\sim 8 \mu \mathrm{m}$ after fabrication based on the adopted UV photolithography condition [29].

The fabricated single GaAs/AlGaAs/GaAs nanowire detectors were finally characterized using a standard THz-TDS system [29, 30] to investigate their $\mathrm{THz}$ response properties. Figure $2(\mathrm{~b})$ shows a schematic of the $\mathrm{THz}$ detection mechanism using a single nanowire photoconductive $\mathrm{THz}$ detector in THz-TDS. Details of the system are shown in Fig. 2(c), where a femtosecond laser with a pulse duration of $\sim 80$ fs and a repetition rate of $84.5 \mathrm{MHz}$ at a central wavelength of $800 \mathrm{~nm}$ was split into two beams. One beam was used to excite a $400-\mu \mathrm{m}$ gap semi-insulatingGaAs photoconductive $\mathrm{THz}$ emitter (which was biased with a square wave of $\pm 400 \mathrm{~V}$ amplitude at $17 \mathrm{kHz}$ ). The other beam was directly used to excite the detector sample with a fluence of $1.9 \mathrm{~nJ} /$ $\mathrm{cm}^{2} /$ pulse. In the sampling process, both the $\mathrm{THz}$ pulse and the optical pulse were collinearly focused at the center of the detector. As the photon energy is larger than the band gap of the detection material, electron-hole pairs are generated in the gap region of the nanowire under photoexcitation. The alternating electric field of the $\mathrm{THz}$ pulse then separates these electron-hole pairs forming a transient photocurrent across the two electrodes. Since the intensity of the transient photocurrent formed in the detector is related to the strength of the incident $\mathrm{THz}$ electric field, by changing the time delay between the $\mathrm{THz}$ pulse and optical pulse at the detector, the temporal profile of the THz-induced photocurrent can be recorded which relates to the temporal waveform profile of the incident $\mathrm{THz}$ electric field. The THzinduced photocurrent between the two electrodes of a photoconductive detector with respect to the $\mathrm{THz}$ pulse-optical pulse time delay $(\tau)$ can be expressed by [26]

$$
I(\tau) \propto \int_{-\infty}^{+\infty} E_{(\mathrm{THz})}(t) \sigma(t-\tau) \mathrm{d} t,
$$

where $E_{(\mathrm{THz})}(t)$ is the effective electric field of the $\mathrm{THz}$ pulse at the photoconductive detector and $\sigma(\tau)$ is the time dependent photoconductivity of the detection material. When the detection material in the photoconductive detector has a charge-carrier lifetime shorter than the duration of the incident $\mathrm{THz}$ pulse (typically $\sim 1-2 \mathrm{ps}$ ), the conductivity $\sigma(\tau)$ of the detection material in Eq. (1) may be approximated as a $\delta$ function and its measured photocurrent intensity will be directly proportional to the strength of the incident $\mathrm{THz}$ electric field,

$$
I(\tau) \propto E_{(\mathrm{THz})}(t=\tau) .
$$

Such detector is categorized as a "direct sampling" photoconductive detector. When the detection material has a much longer charge-carrier lifetime ( $>100 \mathrm{ps}$ ) than the duration of the $\mathrm{THz}$ pulse, the conductivity $\sigma(\tau)$ of the detection material will be approximated as a unit step function and the detector will be categorized as an "integrating" photoconductive detector, where

$$
I(\tau) \propto \int_{\tau}^{+\infty} E_{(\mathrm{THz})}(t) \mathrm{d} t .
$$

It is noted that the THz electric field $E_{(\mathrm{THz})}(t)$ can be recovered from the $I(\tau)$ data by differentiation with respect to time.

\section{Results and discussion}

For THz-TDS measurements, an $800 \mathrm{~nm}(1.55 \mathrm{eV})$ optical pulse was used to photoexcite the nanowire detectors at a normal angle of incidence. The polarizations of both the optical pulse and the $\mathrm{THz}$ pulse were aligned parallel to the nanowire axis (that is the electrode gap direction in the detector). Since the alloy content of aluminium in the AlGaAs shell is nominally $42 \%$, higher energy $(>1.94 \mathrm{eV})$ is required to photoexcite AlGaAs compared to GaAs. Therefore, it is reasonable to assume that the measured $\mathrm{THz}$-induced photocurrent in the nanowire detector is only generated in the GaAs core and the outer GaAs cap layer. The passivated GaAs core is, no doubt, an integrating detector due to its long photoconductivity lifetime ( 4 ns, see Fig. 11(b)); whereas the highly defective and un-passivated GaAs cap layer is anticipated to be a direct sampling detector due to its ultrashort photoconductivity lifetime (a few ps). Because the $\mathrm{Au}$ electrodes are electrically contacted to both the core and cap of the nanowire in the detector, it is expected that two sampling 
modes will be simultaneously observed in the operation of the GaAs/AlGaAs/GaAs single nanowire detector. Moreover, the bow-tie antenna electrode [29] used in this work has been proven not only to strengthen the incident $\mathrm{THz}$ photocurrent signal significantly, but also to cause distortions in the measured waveform which are different under direct and integrating sampling modes (see Fig. 3(a)). It is anticipated that, by analysis of the proportions of the two sampling modes occurring in the single GaAs/AlGaAs/GaAs nanowire detector, contributions in device performance from the core and the cap can be differentiated. Figure $3(\mathrm{~b}, \mathrm{c})$ shows $\mathrm{THz}$ responses obtained from two respective single GaAs/AlGaAs/GaAs nanowire detectors studied in this work. In contrast to the $\mathrm{THz}$ response from a single core-only InP nanowire detector [29], which exhibits a pure integrating sampling behaviour as shown in Fig. B(d), the response profiles of the single GaAs/AlGaAs/GaAs nanowire detectors do not resemble either of the two mode responses (see Fig. 3.a)), but appear as a combination of both. This strongly suggests that direct and integrating sampling modes occur simultaneously in the GaAs/AlGaAs/GaAs nanowire detector.

We note that the proportions of the two mode responses in a GaAs/AlGaAs/GaAs nanowire are comparable to the ratio of the GaAs cap volume to the total GaAs volume of the nanowire (which are $40-90 \%$ in this study according to the statistical analysis of our TEM cross-sectional data). The non-uniformity in responses for each coreshell-cap nanowire detector (see Fig. B(d, e)) can be attributed to the nanowire-to-nanowire variation in their core and cap volumes in growth. It is worth noting that although the amplitude of the fast decaying component is $61 \%$ of the total photoconductivity signal in the OPTP photoconductivity measurement for the GaAs/AlGaAs/ GaAs nanowires (see Fig. 1(b)) comparable to the ratio of the GaAs cap volume to the total GaAs volume of the nanowire, the OPTP measurement [25] is based on measuring the ensemble of nanowires and thus shows an average value (rather than a case-by-case value obtained in the $\mathrm{THz}$ TDS measurement of a single nanowire detector).

(a)
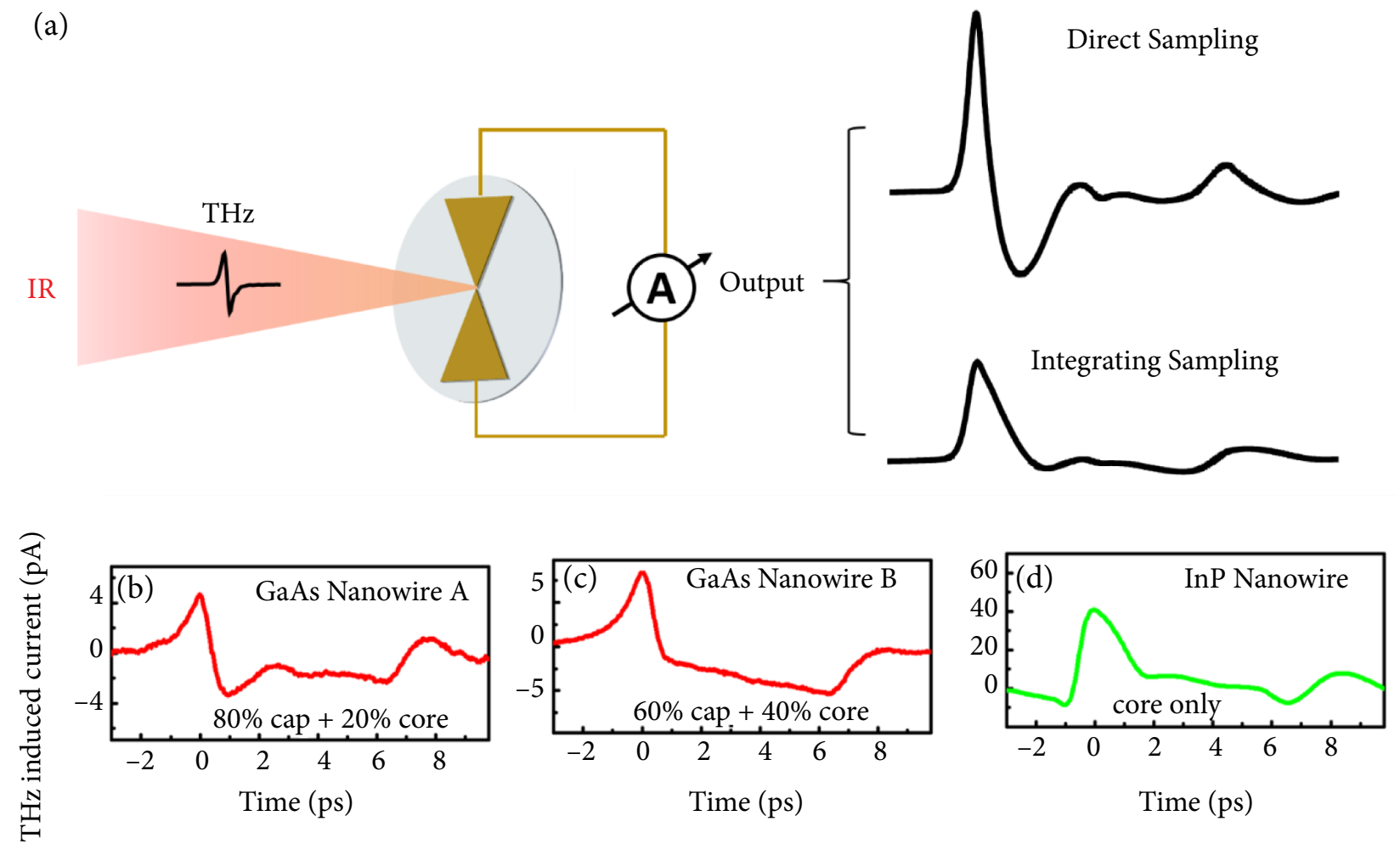

Fig. 3. (a) Simulated time-domain THz responses of bow-tie photoconductive antennas in a THz-TDS system. Simulation details can be found in Ref. [29]. (b-d) Time-domain THz responses measured from two respective bow-tie single GaAs/AlGaAs/AlGaAs core-shell-cap nanowire detectors (red online) and a bow-tie single InP core-only nanowire detector (green online) in our THz-TDS system. 
Most interestingly, we found a single GaAs/ $\mathrm{AlGaAs} / \mathrm{GaAs}$ nanowire detector which had predominantly operated under a direct sampling mode [26] as shown in Fig. 4(a) (a red solid line, cap volume $>90 \%$ in total). Most importantly, this suggests that by accurate control of the volume ratio between the GaAs cap layer and GaAs core we can control the dominant sampling mode in the single GaAs/AlGaAs/GaAs core-shell-cap nanowire detector. It is difficult to achieve direct sampling detectors using bare GaAs nanowires, due to the large surface scattering that limits the carrier mobility in bare GaAs nanowires; therefore, tuning the GaAs capping volume provides a perfect way to produce a direct sampling
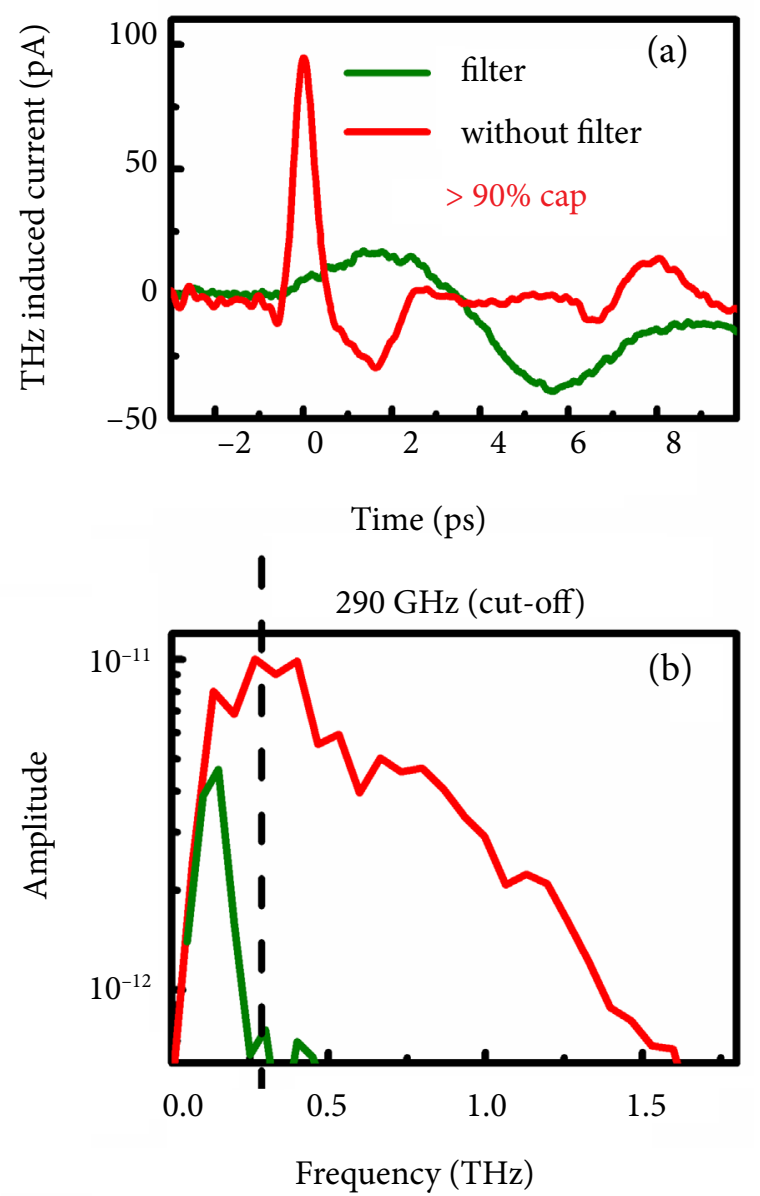

Fig. 4. Performance of a single GaAs/AlGaAs/GaAs core-shell-cap nanowire detector (quasi-direct type). (a) Raw time-domain $\mathrm{THz}$ responses obtained from the nanowire detector without (red online) and with a low-pass filter (green online) in the THz-TDS system. (b) Amplitude spectrum of $\mathrm{THz}$ responses in (a). Black dashed line: cut-off frequency at $290 \mathrm{GHz}$ for the low-pass filter. mode. In fact, this direct sampling is the preferred mechanism for $\mathrm{THz}$ photoconductive detection, as it can greatly simplify the analysis technique [31] reducing any random errors introduced by data processing leading to an improved signal-to-noise ratio [32]. To assess the performance of this quasi-direct single GaAs/AlGaAs/GaAs nanowire detector, a $290 \mathrm{GHz}$ low-pass filter was inserted into the $\mathrm{THz}$ path. Figure 4 shows the $\mathrm{THz}$ responses from the quasi-direct single GaAs/AlGaAs/GaAs nanowire detector without (red) and with (dark green) the presence of the low-pass filter. The data was processed by approximating the response as direct sampling. It can be seen that the cut-off edge is observed at $290 \mathrm{GHz}$ as expected and the transmitted signal amplitude is around $70 \%$ which is in a good agreement with literature [24, 30. This indicates that a quasi-direct single nanowire photoconductive $\mathrm{THz}$ detector was successfully realized through the use of single GaAs based core-shellcap nanowires.

\section{Conclusions}

Two sampling modes (direct and integrating sampling) occur simultaneously in the operation of bow-tie single core-shell-cap nanowire photoconductive $\mathrm{THz}$ detectors. These modes can be attributed to the contributions from the GaAs cap and the GaAs core, respectively, leading to a complex $\mathrm{THz}$ response. By calculating the proportions of the two sampling mode responses in these detectors, the ratio of the GaAs cap volume to the GaAs core volume can be evaluated quantitively. When the contribution of the outer GaAs cap exceeds $90 \%$ of the response, it is reasonable to approximate this response as direct sampling. Such a direct-type photoconductive nanowire detector is highly desired, as it requires simpler data processing.

\section{Acknowledgements}

The Australian Research Council (ARC) and the Engineering and Physical Sciences Research Council (EPSRC) are acknowledged for their financial supports and the authors also thank the use of facilities in the Centre for Advanced Microscopy (AMMRF node), the Australian National Fabrication Facility (ACT node) and the National Computational Infrastructure (NCI) for this work. 


\section{References}

[1] B. Ferguson and X.-C. Zhang, Materials for terahertz science and technology, Nat. Mater. 1(1), 26 (2002).

[2] M. Naftaly and R.E. Miles, Terahertz time-domain spectroscopy for material characterization, Proc. IEEE 95(8), 1658-1665 (2007).

[3] C. Jansen, S. Wietzke, O. Peters, M. Scheller, N. Vieweg, M. Salhi, N. Krumbholz, C. Jördens, T. Hochrein, and M. Koch, Terahertz imaging: applications and perspectives, Appl. Opt. 49(19), E48-E57 (2010).

[4] E.V. Yakovlev, K.I. Zaytsev, I.N. Fokina, V.E. Karasik, and S.O. Yurchenko, Nondestructive testing of polymer composite materials using $\mathrm{THz}$ radiation, J. Phys. Conf. Ser. 486(1), 012008 (2014).

[5] S.F. Federici, B. Schulkin, F. Huang, D. Gary, B. Barat, F. Oliveira, and D. Zimdars, THz imaging and sensing for security applications - explosives, weapons and drugs, Semicond. Sci. Technol. 20(7), S266 (2005).

[6] X. Yang, X. Zhao, K. Yang, Y. Liu, Y. Liu, W. Fu, and Y. Luo, Biomedical applications of terahertz spectroscopy and imaging, Trends Biotechnol. 34(10), 810-824 (2016).

[7] S.S. Dhillon, M.S. Vitiello, E.H. Linfield, A.G. Davies, M.C. Hoffmann, J. Booske, C. Paoloni, M. Gensch, P. Weightman, G.P. Williams, et al., The 2017 terahertz science and technology roadmap, J. Phys. D 50(4), 043001 (2017).

[8] D.H. Auston, Picosecond optoelectronic switching and gating in silicon, Appl. Phys. Lett. 26(3), 101-103 (1975).

[9] M. Hangyo, M. Tani, and T. Nagashima, Terahertz time-domain spectroscopy of solids: a review, Int. J. Infrared Millimeter Waves 26(12), 1661-1690 (2005).

[10]D.H. Auston, K.P. Cheung, and P.R. Smith, Picosecond photoconducting Hertzian dipoles, Appl. Phys. Lett. 45(3), 284-286 (1984).

[11]M. Tani, K. Sakai, and H. Mimura, Ultrafast photoconductive detectors based on semi-insulating GaAs and InP, Jpn. J. Appl. Phys. 36(9A), L1175 (1997).
[12]S. Kono, M. Tani, P. Gu, and K. Sakai, Detection of up to $20 \mathrm{THz}$ with a low-temperature-grown GaAs photoconductive antenna gated with $15 \mathrm{fs}$ light pulses, Appl. Phys. Lett. 77(25), 4104-4106 (2000).

[13]A. Takazato, M. Kamakura, T. Matsui, J. Kitagawa, and Y. Kadoya, Detection of terahertz waves using low-temperature-grown InGaAs with $1.56 \mu \mathrm{m}$ pulse excitation, Appl. Phys. Lett. 90(10), 101119 (2007).

[14]E. Castro-Camus, J. Lloyd-Hughes, L. Fu, H.H. Tan, C. Jagadish, and M.B. Johnston, An ion-implanted InP receiver for polarization resolved terahertz spectroscopy, Opt. Express 15(11), 7047-7057 (2007).

[15]P. Parkinson, H.J. Joyce, Q. Gao, H.H. Tan, X. Zhang, J. Zou, C. Jagadish, L.M. Herz, and M.B. Johnston, Carrier lifetime and mobility enhancement in nearly defect-free core-shell nanowires measured using time-resolved terahertz spectroscopy, Nano Lett. 9(9), 3349-3353 (2009).

[16] H.J. Joyce, C.J. Docherty, Q. Gao, H.H. Tan, C. Jagadish, J. Lloyd-Hughes, L.M. Herz, and M.B. Johnston, Electronic properties of GaAs, InAs and InP nanowires studied by terahertz spectroscopy, Nanotechnology 24(21), 214006 (2013).

[17]H.J. Joyce, S.A. Baig, P. Parkinson, C.L. Davies, J.L. Boland, H.H. Tan, C. Jagadish, L.M. Herz, and M.B. Johnston, The influence of surfaces on the transient terahertz conductivity and electron mobility of GaAs nanowires, J. Phys. D 50(22), 224001 (2017).

[18]N. Jiang, Q. Gao, P. Parkinson, J. Wong-Leung, S. Mokkapati, S. Breuer, H.H. Tan, C.L. Zheng, J. Etheridge, and C. Jagadish, Enhanced minority carrier lifetimes in GaAs/AlGaAs core-shell nanowires through shell growth optimization, Nano Lett. 13(11), 5135-5140 (2013).

[19]H.J. Joyce, Q. Gao, H.H. Tan, C. Jagadish, Y. Kim, $\mathrm{X}$. Zhang, Y. Guo, and J. Zou, Twin-free uniform epitaxial GaAs nanowires grown by a two-temperature process, Nano Lett. 7(4), 921-926 (2007).

[20]J.L. Boland, A. Casadei, G. Tütüncüoglu, F. Matteini, C.L. Davies, F. Jabeen, H.J. Joyce, L.M. Herz, A. Fontcuberta i Morral, and M.B. Johnston, Increased photoconductivity lifetime in GaAs 
nanowires by controlled n-type and p-type doping, ACS Nano 10(4), 4219-4227 (2016).

[21]T. Burgess, D. Saxena, S. Mokkapati, Z. Li, C.R. Hall, J.A. Davis, Y. Wang, L.M. Smith, L. Fu, and P. Caroff, Doping-enhanced radiative efficiency enables lasing in unpassivated GaAs nanowires, Nat. Commun. 7, 11927 (2016).

[22]J.L. Boland, S. Conesa-Boj, P. Parkinson, G. Tütüncüoglu, F. Matteini, D. Rüffer, A. Casadei, F. Amaduzzi, F. Jabeen, and C.L. Davies, Modulation doping of GaAs/AlGaAs core-shell nanowires with effective defect passivation and high electron mobility, Nano Lett. 15(2), 1336-1342 (2015).

[23]N. Jiang, P. Parkinson, Q. Gao, S. Breuer, H.H. Tan, J. Wong-Leung, and C. Jagadish, Long minority carrier lifetime in Au-catalyzed GaAs/ $\mathrm{Al}_{x} \mathrm{Ga}_{1-x}$ As core-shell nanowires, Appl. Phys. Lett. 101(2), 023111 (2012).

[24]K. Peng, P. Parkinson, L. Fu, Q. Gao, N. Jiang, Y.N. Guo, F. Wang, H.J. Joyce, J.L. Boland, H.H. Tan, C. Jagadish, and M.B. Johnston, Single nanowire photoconductive terahertz detectors, Nano Lett. 15(1), 206-210 (2015).

[25]H.J. Joyce, P. Parkinson, N. Jiang, C.J. Docherty, Q. Gao, H.H. Tan, C. Jagadish, L.M. Herz, and M.B. Johnston, Electron mobilities approaching bulk limits in "surface-free" GaAs nanowires, Nano Lett. 14(10), 5989-5994 (2014).

[26]E. Castro-Camus, L. Fu, J. Lloyd-Hughes, H.H. Tan, C. Jagadish, and M.B. Johnston, Photoconductive response correction for detectors of terahertz radiation, J. Appl. Phys. 104(5), 053113 (2008).

[27]K. Peng, P. Parkinson, L. Fu, Q. Gao, N. Jiang, Y.N. Guo, F. Wang, H.J. Joyce, J.L. Boland,
M.B. Johnston, H.H. Tan, and C. Jagadish, Single GaAs/AlGaAs nanowire photoconductive terahertz detectors, in: Proceedings of the 2014 Conference on Optoelectronic and Microelectronic Materials \& Devices (COMMAD) (Institute of Electrical and Electronics Engineers (IEEE), Piscataway, NJ, 2014) pp. 221-222.

[28]H.J. Joyce, J.L. Boland, C.L. Davies, S.A. Baig, and M.B. Johnston, A review of the electrical properties of semiconductor nanowires: insights gained from terahertz conductivity spectroscopy, Semicond. Sci. Technol. 31 (10), 103003 (2016).

[29]K. Peng, P. Parkinson, J.L. Boland, Q. Gao, Y.C. Wenas, C.L. Davies, Z.Y. Li, L. Fu, M.B. Johnston, H.H. Tan, and C. Jagadish, Broadband phase-sensitive single InP nanowire photoconductive terahertz detectors, Nano Lett. 16(8), 4925-4931 (2016).

[30] K. Peng, P. Parkinson, Q. Gao, J.L. Boland, Z.Y. Li, F. Wang, S. Mokkapati, L. Fu, M.B. Johnston, H.H. Tan, and C. Jagadish, Single $\mathrm{n}^{+}-\mathrm{i}-\mathrm{n}^{+}$InP nanowires for highly sensitive terahertz detection, Nanotechnology 28(12), 12520 (2017).

[31]W. Withayachumnankul and M. Naftaly, Fundamentals of measurement in terahertz time-domain spectroscopy, J. Infrared Millim. Terahertz Waves 35(8), 610-637 (2014).

[32]E. Castro-Camus, J. Lloyd-Hughes, M. Johnston, M. Fraser, H. Tan, and C. Jagadish, Polarizationsensitive terahertz detection by multicontact photoconductive receivers, Appl. Phys. Lett. 86(25), 254102 (2005).

\title{
APVALKALO IR ŠERDIES INDE்LIŲ ATSKYRIMAS TERAHERCINIAME GaAs ŠERDIES-KEVALO-APVALKALO TIPO VIENO NANOLAIDO DETEKTORIAUS FOTOLAIDUMO ATSAKE
}

\author{
K. Peng a, b. P. Parkinson ${ }^{\text {c }}$, L. Fu a , Q. Gao a, J. Boland ${ }^{\text {b }}$, Y.-N. Guo a, N. Jian ${ }^{\text {a }}$, H.H. Tan ${ }^{\text {a }}$, \\ M.B. Johnston ${ }^{\mathrm{b}}$, C. Jagadish ${ }^{\mathrm{a}}$ \\ ${ }^{a}$ Australijos nacionalinis universitetas, Kanbera, Australija \\ ${ }^{\mathrm{b}}$ Oksfordo universitetas, Oksfordas, Jungtinè Karalystè \\ ' Mančesterio universitetas, Mančesteris, Jungtinè Karalystè
}

